\title{
Etat nutritionnel et reproduction chez la truie allaitante
}

\author{
H. QUESNEL \\ INRA, Agrocampus Rennes, UMR Systèmes d'Elevage, Nutrition Animale et Humaine \\ F-35590 Saint-Gilles \\ Courriel: Helene.Quesnel@rennes.inra.fr
}

\begin{abstract}
On sait depuis longtemps que le déficit nutritionnel des truies en lactation peut provoquer des problèmes de reproduction après le sevrage des porcelets, notamment chez les jeunes femelles. Ce risque a-t-il été aggravé par la sélection des truies sur les performances de reproduction? Et que sait-on des mécanismes physiologiques qui sous-tendent ces interactions nutrition $\mathrm{X}$ reproduction ?
\end{abstract}

Chez les mammifères domestiques, comme chez les humains, la fonction de reproduction peut être altérée quand les besoins en énergie et/ou en protéines ne sont pas satisfaits. Pendant la lactation, bien que les truies soient nourries à volonté ou presque, souvent elles ne consomment pas suffisamment pour compenser les dépenses nutritionnelles élevées liées à la production laitière (Quiniou et al 1998). Le risque de déficit nutritionnel touche surtout les truies primipares qui ont encore des besoins de croissance et présentent un appétit plus faible que celui des truies multipares, malgré une production de lait déjà abondante. A court terme, le déficit nutritionnel pendant la lactation peut altérer les performances de reproduction et notamment, induire un retard d'œstrus après le sevrage, surtout chez les jeunes femelles (Aherne et Kirkwood 1985). A plus long terme, le déficit peut réduire la longévité des truies (Dourmad et al 1994). Dans les années 90, les problèmes de reproduction (anœstrus, échec de gestation, petite portée...) représentaient 30 à $50 \%$ des causes de réformes des truies en élevage (Lucia et al 2000). L'évolution des génotypes porcins pourrait faire craindre une augmentation de l'incidence des problèmes de reproduction. Le développement récent de lignées hyperprolifiques grâce à la sélection et/ou au croisement avec des races asiatiques a accéléré l'accroissement de la prolificité. Celle-ci a augmenté de plus de deux porcelets par portée dans les vingt dernières années et, dans le même temps, la production laitière s'est améliorée de $50 \%$ environ. Malgré cela, les problèmes de reproduction n'ont globalement pas augmenté en France depuis l'introduction des truies hyperprolifiques dans les élevages (Boulot 2004).
Dans une première partie, cette synthèse décrit l'impact du déficit nutritionnel pendant la lactation sur les performances de reproduction et l'activité de l'axe gonadotrope (ou axe hypothalamus-hypophyse-ovaires) chez la truie. L'accent est porté sur les études des dix dernières années, sur des truies issues de races européennes. La seconde partie fait le bilan des connaissances sur les mécanismes physiologiques impliqués dans l'interaction entre statut métabolique et ovulation. Il est généralement admis que l'axe gonadotrope est informé de l'état nutritionnel des animaux via les modifications métaboliques et physiologiques induites par le déficit nutritionnel (Booth 1990, Pettigrew et Tokach 1993, Cosgrove et Foxcroft 1996, Prunier et Quesnel 2000a, b). En élevage, la sous-consommation des truies pendant la lactation induit un déficit énergétique et un déficit protéique. Cette synthèse traite de ces deux aspects, qui en expérimentation peuvent être induits simultanément en limitant l'apport en aliment ou séparément en fournissant aux truies des rations hypoprotéiques ou hypoénergétiques.

\section{1 / Déficit nutritionnel, réserves corporelles et reproduction}

\section{1 / L'impact du déficit nutri- tionnel sur le retour en ostrus après le sevrage}

La truie, comme les autres mammifères, est généralement en anœstrus pendant l'allaitement qui est relativement court. La sécrétion du GnRH est en effet inhibée par des neurotransmetteurs libérés dans le système nerveux central lors de la tétée. La sécrétion des hormones gonadotropes FSH et LH est donc réduite (Britt et al 1985, Varley et Foxcroft 1990, Quesnel et Prunier 1995). Le sevrage des porcelets, après 3 à 5 semaines de lactation, lève cette inhibition et la fréquence des pulses de LH s'accélère immédiatement. Elle stimule la croissance préovulatoire des follicules qui mène à l'œstrus et l'ovulation. La durée de l'intervalle sevrageœstrus (ISO) est influencée par le profil de sécrétion de $\mathrm{LH}$ pendant la lactation et au moment du tarissement (Shaw et Foxcroft 1985). La durée moyenne et la variabilité de l'ISO ont été considérablement réduites au cours des vingt dernières années (Soede et al 2000). De nos jours, la majorité des truies reviennent en chaleur entre 4 et 7 jours après le sevrage des porcelets. Malgré ces progrès, la variabilité de l'ISO reste un problème en élevage car elle perturbe la conduite en bandes des truies. Aussi la mise à la reproduction est-elle associée à une utilisation accrue des traitements hormonaux qui permettent d'induire l'ovulation. Dans certains élevages, en effet, ces traitements sont appliqués systématiquement aux truies primipares le jour du sevrage. Cette pratique pourrait ne plus être autorisée dans le cadre du développement de systèmes d'élevage durables en Europe, ce qui pose le problème de la maîtrise de l'état nutritionnel des animaux. Enfin, il faut savoir qu'un allongement même minime de l'ISO est associé à une baisse notable des performances de reproduction ultérieures, notamment du taux de gestation et de la taille de la portée (Vesseur et al 1994, Le Cozler et al 1997). 
Lorsque les truies sont soumises à un rationnement alimentaire pendant la lactation, l'apparition de l'œstrus après le sevrage des porcelets peut être retardée chez les truies en première (figure 1) ou deuxième portée (Kirkwood et al 1987, 1990, Baidoo et al 1992). Le retard d'œstrus est observé si l'énergie métabolisable fournie par l'aliment couvre moins de $60 \%$ des besoins énergétiques totaux (entretien et production de lait) chez les truies rationnées, contre 85 à $100 \%$ chez les truies témoins (Prunier et Quesnel 2000a). Un allongement de l'ISO, de même ampleur, est également observé lorsque le rationnement est ciblé, à savoir que seul l'apport en énergie ou seul l'apport en protéines est réduit (figure 1). Pendant la lactation, et juste après le sevrage des porcelets, la fréquence des pulses de LH est plus faible chez les truies rationnées que chez les truies témoins, que le rationnement soit total, énergétique ou protéique (figure 2). Or la fréquence des pulses de LH est, généralement, le reflet de la fréquence des pulses de GnRH (Caraty et al 2001). Le déficit énergétique et/ou protéique induit donc un retard d'œstrus en amplifiant l'inhibition de la sécrétion du GnRH pendant la lactation.

L'impact du déficit protéique sur la sécrétion de LH et l'ISO dépend du
Figure 2. Conséquence d'un rationnement en aliment (• ○), en énergie métabolisable $(\square)$ ou en protéines $(\Delta \triangle)$ pendant la lactation sur la fréquence des pulses de $\mathrm{LH}$ en fin de lactation chez des truies primipares.

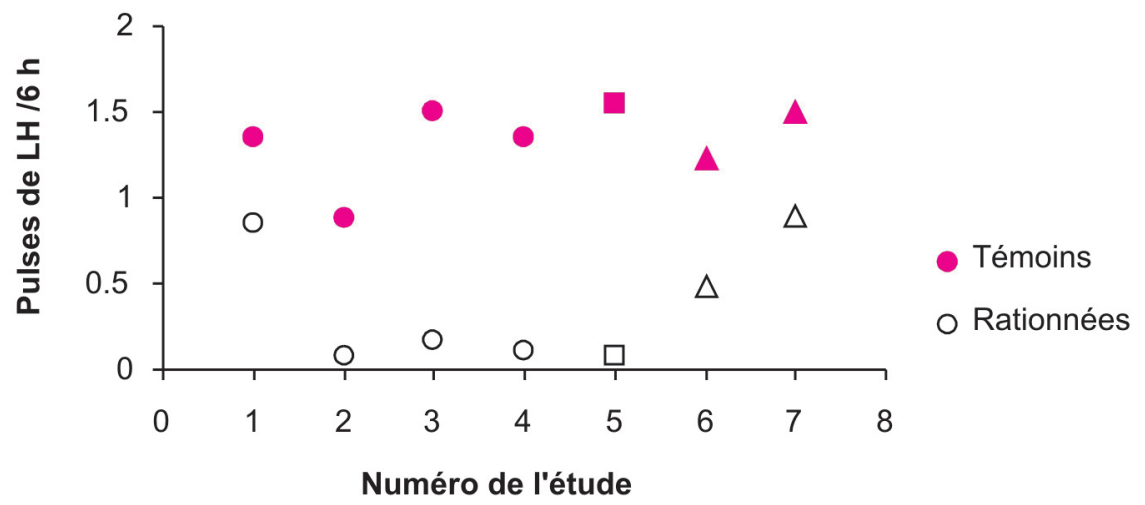

Références : 1. Mullan et al 1991 (90/50), 2. Zak et al 1997a (80/45), 3. Quesnel et al 1998a (90/50), 4. Zak et al 1998 (85/50), 5. Koketsu et al 1998 (70/40), 6. Jones et Stahly 1999 (120/40), 7. Yang et al 2000b (85/40).

Entre parenthèses : l'apport en énergie ou protéines exprimé en pourcentage des besoins pour les truies témoins et rationnées respectivement (voir légende de la figure 1). niveau d'apport en énergie. Augmenter l'apport protéique permet de compenser partiellement un déficit énergétique modéré (60 - $70 \%$ des besoins énergétiques) et peut restaurer, au moins partiellement, l'ISO des truies primipares (King et Williams 1984, Tokach et al 1992a). Cependant cela est sans effet si le déficit énergétique est plus sévère (50\% des besoins). D'un point de vue nutritionnel cette réponse est logique, puisque, en situation de déficit énergé-
Figure 1. Conséquence d'un rationnement en aliment (• ○), en énergie métabolisable $(\square)$ ou en protéines $(\Delta \triangle)$ pendant la lactation sur la durée de l'intervalle sevrage œstrus (ISO) chez des truies primipares $\left({ }^{*}: P<0,05\right)$.

\section{Rationnement en :}

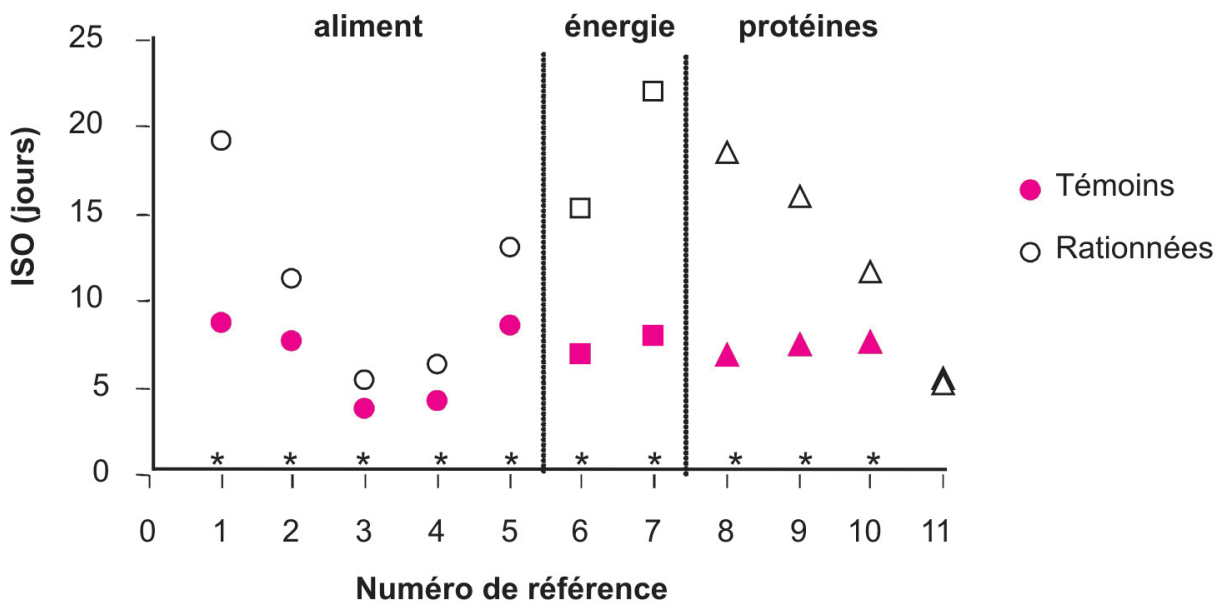

Références : 1. Mullan et al 1991 (90/50), 2. Prunier et al 1993 (80/50), 3. Zak et al 1997a (80/45), 4. Zak et al $1998(85 / 50)$, 5. Koketsu et al 1998 (70/40), 6. King et Williams $1984(100 / 45)$, 7. Koketsu et al 1996a (75/35), 8. King et Williams 1984 (100/40), 9. King et Martin 1989 (84/40), 10. Jones et Stahly 1999 (120/40), 11. Mejia-Guadarrama et al 2002 (100/47).

Entre parenthèses : l'apport en énergie ou protéines exprimé en pourcentage des besoins pour les truies témoins et rationnées respectivement (les besoins en énergie métabolisable sont estimés d'après une équation proposée par Noblet et al (1990) et les besoins en lysine sont évalués pour une mobilisation nulle des protéines corporelles, d'après une équation proposée par Dourmad et al (1998). tique, les protéines sont catabolisées pour servir de substrat énergétique. Inversement, augmenter l'apport en énergie sera bénéfique seulement si l'apport en protéines n'est pas limitant (Prunier et Quesnel 2000a).

Au-delà de la deuxième portée, l'ISO dépend peu de l'état nutritionnel des truies. En effet, un rationnement alimentaire même sévère est sans conséquence sur l'ISO ou la sécrétion de LH (Hughes et al 1993, Varley et al 1996). Les truies âgées sont donc non seulement moins sujettes au déficit nutritionnel que les jeunes femelles, mais aussi moins sensibles.

\section{2 / L'impact du déficit nutri- tionnel sur le taux d'ovulation et la survie embryonnaire}

Les études réalisées dans les dix dernières années sur des truies primipares montrent une répercussion du déficit nutritionnel sur le taux d'ovulation (nombre d'ovocytes émis) au premier œstrus post-sevrage. Une réduction moyenne de 2 à 5 ovocytes est observée après un rationnement alimentaire (Zak et al 1997a, van den Brand et al 2000) ou protéique (Mejia-Guadarrama et al 2002, Quesnel et al 2005b) pendant la lactation. Cette baisse du taux d'ovulation ne semble pas liée au profil de sécrétion de $\mathrm{LH}$ en fin de lactation. Il faut noter que dans ces études récentes, le déficit nutritionnel induit n'engendre qu'un retard marginal de l'œstrus après le sevrage $(+1$ ou 2 jours, Zak et al $1997 \mathrm{a}, 1998$ ) voire pas de retard du tout (van den Brand et al 2000, MejiaGuadarrama et al 2002). Cela suggère que lorsque l'ISO est court, les truies 
n'ont pas totalement récupéré de leur état catabolique au moment de la sélection des follicules qui pourraient ovuler. Aussi, les progrès réalisés en élevage sur la maîtrise de l'ISO pourraient s'accompagner d'une détérioration d'autres paramètres de reproduction dans les situations nutritionnelles limitantes.

Le déficit nutritionnel, notamment en fin de lactation, peut également altérer la survie embryonnaire au cours de la gestation (Zak et al 1997a). Cette altération peut être liée à la moindre qualité ovocytaire et folliculaire et/ou à des perturbations de l'environnement hormonal intra-utérin. En effet, il a été montré que l'aptitude des ovocytes à maturer in vitro et l'aptitude du liquide folliculaire à stimuler la maturation ovocytaire sont réduites lorsque les follicules et les ovocytes prélevés 3 ou 4 jours après le sevrage sont issus de truies rationnées pendant la lactation (rationnement alimentaire : Zak et al 1997b, ou protéique : Yang et al 2000a). Cependant, les mécanismes physiologiques par lesquels le déficit nutritionnel altère la survie des embryons ne seront pas abordés dans cet article (voir revue de Foxcroft 1997).

En élevage, la taille de la portée pourra être touchée si la réduction du taux d'ovulation se cumule à une moindre survie embryonnaire, celle-ci pouvant résulter de l'état catabolique des truies mais aussi de facteurs environnementaux (conditions sanitaires, températures ambiantes...).

\section{3 / Existe-t-il une phase cri- tique pendant la lactation?}

Dans une étude de terrain portant sur 24000 mises bas, Koketsu et al (1996b) mettent en évidence un allongement de l'ISO pour les truies, tous rangs de portée confondus, qui consomment peu pendant les trois semaines de lactation ou juste pendant la première semaine. L'ISO des truies primipares est également prolongé (d'un jour en moyenne) si les truies présentent une baisse de consommation marquée (- $25 \%$ au moins) pendant seulement quelques jours. Compte tenu des pratiques des éleveurs, ces trois catégories de truies présentent un taux de réforme pour anœstrus supérieur à la moyenne (Koketsu et al 1996b). Ces mêmes auteurs ont montré qu'une seule semaine de rationnement énergétique, sur une lactation de 3 semaines, suffit à inhiber la sécrétion de LH et à retarder
Tableau 1. Performances de reproduction après le sevrage de truies primipares soumises à un rationnement en protéines (lysine) pendant la lactation (Quesnel et al 2005b).

\begin{tabular}{|l|c|c|c|c|}
\hline Lot & 180CP & 180LP & 240LP & Stat. \\
\hline Poids à la mise bas, $\mathrm{kg}$ & $182,6 \mathrm{a}$ & $181,1 \mathrm{a}$ & $238,2 \mathrm{~b}$ & $P<0,001$ \\
Lysine ingérée, $\mathrm{g} / \mathrm{j}$ & $39,4 \mathrm{a}$ & $21,6 \mathrm{~b}$ & $16,0 \mathrm{c}$ & $P<0,001$ \\
Energie métabolisable ingérée, MJ/j & $53,1 \mathrm{a}$ & $54,8 \mathrm{a}$ & $45,6 \mathrm{~b}$ & $P<0,05$ \\
\hline Truies en chaleurs dans les 9 jours après & & & & \\
le sevrage : & & & & \\
Proportion & $11 / 12 \mathrm{a}$ & $7 / 12 \mathrm{~b}$ & $12 / 14 \mathrm{a}$ & $P=0,03$ \\
Nombre de corps jaunes au premier œstrus & $17,1 \mathrm{a}$ & $14,0 \mathrm{~b}$ & $15,6 \mathrm{ab}$ & $P=0,05$ \\
\hline
\end{tabular}

a, b, c, intraligne : $P<0,05$.

l'œstrus après le sevrage, que le rationnement soit imposé en première, deuxième ou troisième semaine de lactation (Koketsu et al 1996a). Un rationnement alimentaire imposé pendant les trois premières semaines de lactation ou pendant la quatrième (et dernière) semaine allonge l'ISO et réduit le taux d'ovulation (Zak et al 1997a). Dans ces conditions, il est donc difficile d'identifier une période critique de la lactation pendant laquelle l'impact du déficit nutritionnel sur l'ISO serait accru. En ce qui concerne la survie embryonnaire, en revanche, la dernière semaine de lactation pourrait être critique (Zak et al 1997a et b). L'impact du déficit sur la reproduction sera d'autant plus fort en fin de lactation que les réserves corporelles auront été largement mobilisées.

Limiter la période de déficit alimentaire en réduisant la durée d'allaitement n'est cependant pas nécessairement favorable aux performances de reproduction après le tarissement. Ainsi, dans les élevages français, les durées moyennes de l'intervalle sevrage œstrus ou sevrage - saillie fécondante et leur variabilité sont minimales pour les durées d'allaitement supérieures à 25 jours (Dagorn et al 1996). Ceci s'explique physiologiquement par le fait que l'inhibition de l'axe gonadotrope est très intense pendant les quinze premiers jours de lactation puis s'atténue progressivement en troisième et quatrième semaines de lactation (Quesnel et Prunier 1995).

\section{4 / Le rôle des réserves corpo- relles}

Il a été montré que la durée de l'ISO est positivement liée à l'importance de la mobilisation des réserves corporelles pendant la lactation, aussi bien en terme de poids vif, de lard dorsal ou de masse protéique, mais est négativement liée à la quantité de réserves corporelles présentes au sevrage (King 1987, Whittemore et Morgan 1990). Ainsi, la fonction du poids des truies suggère que l'ISO est optimal pour des poids au sevrage supérieurs à $150 \mathrm{~kg}$ (Williams et Mullan 1989) ou à $180-190 \mathrm{~kg}$ (Charette et al 1995). Enfin, certains auteurs montrent que la masse protéique perdue pendant la lactation ou présente au sevrage est un meilleur prémasse lipidique perdus ou restants au sevrage (King 1987, Charrette et al 1985).

Nous avons récemment montré le rôle protecteur, au moins partiel, des réserves corporelles contre les effets du déficit protéique. Un rationnement protéique imposé pendant la lactation à des truies primipares pesant en moyenne 180 ou $240 \mathrm{~kg}$ à la mise bas altère significativement le retour en œstrus des truies légères mais pas celui des truies lourdes (tableau 1, Quesnel et al 2005b). Il induit aussi une réduction du taux d'ovulation au premier œstrus après le sevrage, plus marquée chez les truies légères que chez les truies lourdes. Dans une expérience similaire, des truies pesant en moyenne $165 \mathrm{~kg}$ à la mise bas présentent des ovaires moins développés au sevrage que des truies de $190 \mathrm{~kg}$, malgré un déficit protéique de même ampleur dans les deux lots de truies (Clowes et al 2003a). Le rôle des réserves corporelles avait déjà été montré en cas de déficit nutritionnel global (Mullan et Williams 1989). Un poids vif élevé à la mise bas permettrait donc aux truies de mieux supporter un déficit nutritionnel pendant la lactation sans que leurs performances de reproduction ne soient affectées. En pratique, 1'alimentation des truies pendant la gestation devrait donc favoriser la constitution des réserves corporelles. Néanmoins, comme l'appétit des truies en lactation est inversement lié à leur adiposité à la mise bas, il semble soureprésentation graphique de l'ISO en dicteur de l'ISO que le poids vif ou la 
haitable de favoriser le dépôt protéique pendant la gestation sans exagérer le dépôt lipidique.

Cet impact des variations et du niveau des réserves corporelles sur la reproduction est cohérent avec les observations selon lesquelles les truies primipares sont dans l'ensemble plus sensibles que les truies multipares.

\section{2 / Les mécanismes physio- logiques}

Nous avons vu que le déficit nutritionnel amplifie l'anœstrus en inhibant la sécrétion du $\mathrm{GnRH}$, ce qui retarde la décharge pré-ovulatoire de LH, tandis que son impact sur le taux d'ovulation n'est pas nécessairement lié à l'altération des sécrétions gonadotropes. Suppression de l'ovulation et réduction $\mathrm{du}$ taux d'ovulation impliquent donc des mécanismes différents, agissant au niveau hypothalamo-hypophysaire pour le premier et au niveau ovarien pour le second. Ces deux aspects seront donc traités séparément. Nous dissocierons aussi le déficit protéique du déficit énergétique, bien qu'ils aient tous deux le même impact sur l'axe gonadotrope. En effet, si certaines conséquences métaboliques sont communes aux deux types de déficits, d'autres leur sont propres (voir $\S 2.1 \mathrm{~b}$ ). Il est donc possible que des voies d'action différentes existent pour les deux situations.

\section{1 / Les mécanismes physiolo- giques agissant au niveau cen- tral}

\section{a) En situation de déficit énergétique}

De nombreuses synthèses sont disponibles sur les relations entre métabolisme énergétique et reproduction chez les mammifères (Downing et Scaramuzzi 1991, Monget et al 2001, Wade et Jones 2003) et un consensus existe sur les principales voies d'action (figure 3). La consommation volontaire d'aliments module la disponibilité en substrats oxydables issus de la digestion, à savoir le glucose, les acides gras et les Acides Aminés (AA). Cette disponibilité en substrat module les sécrétions hormonales (insuline, leptine, insulin-like growth factor -IGF-I-) ou l'activité neuronale (nerf vague...) au niveau de différents organes ou tissus, notamment le pancréas, le tissu adipeux, le foie, l'aire post-trema dans le cerveau. Enfin, les substrats disponibles et/ou les hormones et neurotransmetteurs mis en jeu modulent l'activité des neurones au GnRH soit en agissant directement dans la zone qui contient ces neurones, soit en agissant au niveau de l'aire post-trema et de la transmission de l'information entre aire posttrema et neurones à GnRH (figure 3). En particulier, ces substrats ou hormones peuvent moduler la sensibilité hypothalamique au rétrocontrôle négatif qu'exerce l'œstradiol ovarien sur l'activité des neurones à GnRH. Comme le rétrocontrôle exercé par l'œstradiol varie d'intensité et de sens (négatif ou positif) au cours du cycle œstrien et selon le stade physiologique, on peut concevoir que ces interactions varient selon les femelles étudiées (prépubères, cycliques, ovariectomisées, allaitant...).

Ce schéma général nécessite d'être précisé, espèce par espèce. Premièrement, parce qu'il est bâti en partie sur des résultats obtenus chez les rongeurs. Vu l'extrême sensibilité des rongeurs au rationnement alimentaire et leur faible adiposité, l'extrapolation des résultats aux mammifères domestiques et notamment à la truie peut ne pas être correcte (Clarke et Henry 1999). Deuxièmement, il repose aussi sur des résultats obtenus dans des situations extrêmes, par exemple de désactivation de gènes ou de pathologie (diabète, obésité), surtout pour l'étude du rôle de l'insuline et de la leptine. Il reste souvent à déterminer si des variations physiologiques de ces hormones peuvent aussi affecter l'activité hypothalamique.

Chez la truie, le rationnement énergétique pendant la lactation induit la mobilisation des lipides du tissu adipeux, et donc une augmentation des concentrations circulantes des Acides Gras Libres (AGL), et s'accompagne d'une mise à contribution des masses protéiques. Il induit une réduction des concentrations plasmatiques moyennes d'insuline, de glucose, d'IGF-I et de leptine.

\section{- Le glucose et l'insuline}

Le porc étant un animal monogastrique, l'énergie métabolisable est essentiellement fournie sous forme de glucose. Le transporteur de glucose dépendant de l'insuline (GLUT4) et le récepteur à l'insuline ont été localisés dans les zones du cerveau qui contiennent les neurones au GnRH (Monget et Martin 1997). La faible insulinémie due à la restriction nutritionnelle doit donc limiter l'entrée du glucose dans ces cellules. Par ailleurs, la présence de cellules sensibles à la quantité de glucose disponible a été mise en évidence dans l'aire post-trema située dans le système nerveux central. Chez la ratte et la femelle de hamster, une restriction en glucose induite expérimentalement par injection d'un inhibiteur de la glycolyse, le 2-déoxy-D-glucose, stoppe la cyclicité des femelles, sauf si la zone contenant ces cellules a été détruite au préalable (Wade et al 1996). Chez la cochette prépubère, le rôle du glucose dans la sécrétion de LH est étayé. Une injection de glucose à des cochettes rationnées induit une augmentation de la fréquence des pulses de LH, augmentation similaire à celle observée chez ces cochettes après réalimentation (Booth 1990, figure 4). A l'inverse,

Figure 3. Représentation schématique des voies d'action par lesquelles la disponibilité en substrats oxydables affecterait la sécrétion du GnRH (adapté de Wade et al 1996, Monget et al 2001, Wade et Jones 2003).

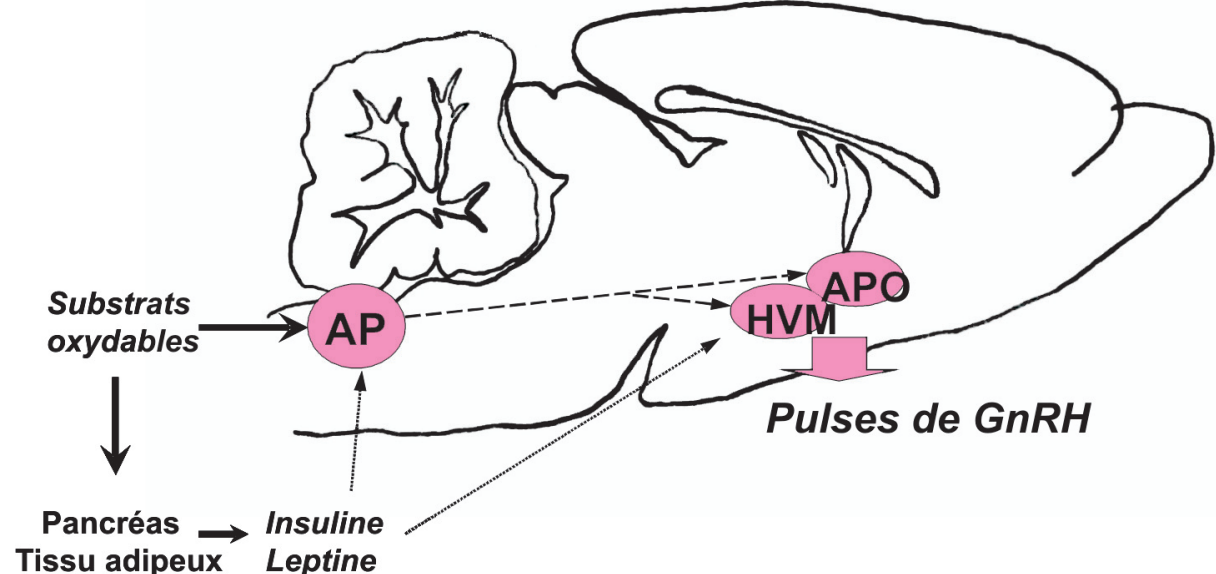

La disponibilité en substrats est détectée par l'aire post-trema (AP) et l'information est transmise à la zone contenant les neurones au GnRH et les neurones sensibles aux stéroïdes sexuels (hypothalamus ventro-médian - HVM- et aire pré-optique - APO). La transmission de l'information ferait intervenir différentes voies d'action et de nombreux neurotransmetteurs, non décrits ici. Insuline et leptine pourraient intervenir à différents niveaux. 
Figure 4. Effet d'une injection ou d'une restriction en glucose sur la fréquence des pulses de LH chez des cochettes prépubères.

A. Injection de glucose

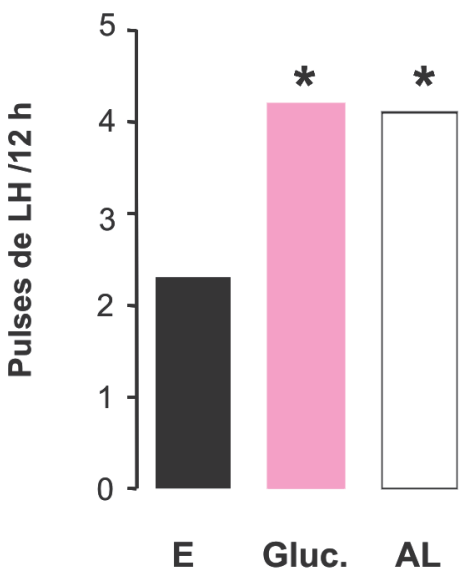

B. Restriction en glucose

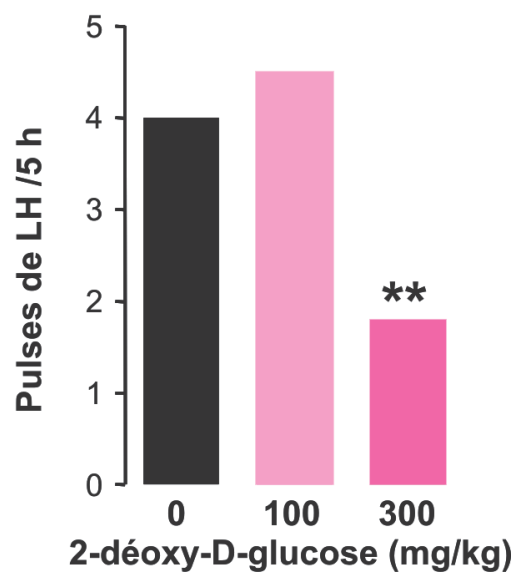

A. Pendant 7 jours les cochettes reçoivent une ration alimentaire quotidienne qui couvre les besoins d'entretien. Le $8^{\mathrm{e}}$ jour, un tiers des femelles sont nourries à volonté (AL). Les autres cochettes sont maintenues à l'entretien $(E)$ et certaines d'entre elles reçoivent des injections i.v. de glucose (Gluc.). La quantité de glucose injectée apporte la même quantité d'énergie que le surplus d'aliment consommé par les truies AL (Booth 1990). B. Les cochettes, ovariectomisées, sont nourries de façon standard et reçoivent une injection i.v. de 2-déoxy-D-glucose, un inhibiteur de la glycolyse qui induit un déficit en glucose (Barb et al 2001a).

${ }^{*} \mathrm{P}<0,05,{ }^{* *} \mathrm{P}<0,01$.

l'injection de 2-déoxy-D-glucose diminue par deux la fréquence des pulses de LH (Barb et al 2001a, figure 4). En revanche, chez la truie en lactation, une perfusion de glucose est sans effet sur la sécrétion de LH, malgré une augmentation significative des concentrations plasmatiques de glucose et d'insuline pendant le traitement (Tokach et al 1992b).

Le rôle primordial de l'insuline sur la sécrétion des hormones gonadotropes a été démontré chez la souris. L'inactivation spécifique du récepteur à l'insuline dans le système nerveux central, par la technique du knock-out, induit une obésité, une baisse de fertilité et une altération de la folliculogénèse due à un dysfonctionnement hypothalamique (Brüning et al 2000). Chez des cochettes prépubères ou cycliques, l'administration d'insuline par voie intraveineuse ou sous-cutanée n'influence pas la sécrétion de LH ou la stimule (Cox et al 1987, Matamoros et al 1991). Chez des truies ovariectomisées, l'injection d'insuline dans le troisième ventricule augmente la sécrétion de LH (Cox et al 1990).

La sécrétion de glucose et d'insuline dépend beaucoup de l'apport d'énergie par l'aliment, mais également de la source énergétique. Chez des truies en lactation, à apport énergétique égal, un aliment enrichi en amidon induit un pic post-prandial d'insuline et de glucose plus élevé qu'un aliment enrichi en lipi- des (Kemp et al 1995, van den Brand et al 2000). L'aliment enrichi en amidon augmente la fréquence des pulses de LH à 7 jours de lactation mais pas audelà, et n'a pas d'impact sur la durée de l'intervalle sevrage-œstrus (Kemp et al 1995, van den Brand et al 2000). Distribuer cet aliment aux truies allaitantes en élevage ne permettrait donc pas de réduire le risque d'œstrus tardif.

- L'IGF-I (Insulin-like Growth Factor-I)

Des injections intracérébroventriculaires d'IGF-I augmentent la sécrétion de LH chez le rat (Monget et Martin 1997), mais pas chez des cochettes prépubères ovariectomisées (Barb et al 2001b). Quand des cochettes prépubères sont soumises à un rationnement alimentaire, Booth et al (1996) observent que la baisse de LH dans le plasma précède celle d'IGF-I. L'hypothèse d'un rôle direct de l'IGF-I dans l'inhibition du GnRH chez les femelles rationnées n'est pas étayée chez la truie, qu'elle soit en lactation ou cyclique. De plus, l'IGF-I circule essentiellement lié à des protéines de liaison spécifiques (les IGFBP). Ses effets dépendent pour beaucoup de sa biodisponibilité, qui est essentiellement contrôlée par un mécanisme spécifique de protéolyse de ces IGFBP (Le Roith et al 2001). A notre connaissance, l'influence du déficit énergétique sur la biodisponibilité de l'IGF-I n'est pas connue chez la truie en lactation.

\section{- La leptine}

La leptine, hormone essentiellement sécrétée par le tissu adipeux, régule le comportement alimentaire et les dépenses énergétiques (Houseknecht et Portocarro 1998, Barb 1999). Elle a pour caractéristique d'avoir des taux circulants modulés surtout par le niveau d'adiposité et en partie par le bilan énergétique instantané (Barb 1999, Chilliard et al 2001). Les ARN messagers codant pour la forme active du récepteur à la leptine ont été localisés dans l'hypothalamus de nombreuses espèces y compris le porc (Lin et al 2000). Chez les rongeurs et la femme, des arguments existent en faveur d'un rôle de la leptine dans les interactions entre métabolisme et reproduction. Ainsi, la leptine exogène restaure partiellement la sécrétion de LH chez des souris normales soumises à deux jours de jeûne (Ahima et al 1996). Elle stimule la sécrétion de LH et la croissance folliculaire chez des femmes qui présentent une aménorrhée d'origine hypothalamique due à une activité physique intense ou un faible poids, et elle restaure les cycles sexuels chez certaines de ces patientes (Welt et al 2004). Chez la vache et la brebis, la stimulation de l'unité hypothalamo-hypophysaire par la leptine est observée essentiellement chez des femelles qui sont en déficit énergétique profond, induit par un jeûne de 2 ou 3 jours ou par un rationnement de plus longue durée (Barb et Kraeling 2004, Zieba et al 2005). Ceci suggère que la réponse hypothalamique à la leptine dépend du statut métabolique des femelles.

Chez la truie, la leptine ne semble pas servir de signal métabolique en cas de déficit énergétique aigu. Ainsi, le jeûne réduit considérablement les concentrations de leptine sans altérer la sécrétion de LH (Barb et al 2001b). Le rôle de la leptine n'est pas non plus établi dans les situations de déficit énergétique de plus longue durée. En fait, dans l'espèce porcine, la leptine serait plus un médiateur entre statut métabolique au sens large (réserves et bilan instantané) et hypothalamus surtout au moment de la puberté (Barb et Kraeling 2004).

- Le dialogue entre le tractus gastrointestinal et le cerveau

La disponibilité en substrats oxydables est détectée par le cerveau mais aussi par le foie, ce qui intervient dans la régulation de la prise alimentaire via le nerf vague. Des travaux chez les rongeurs suggèrent que l'information transmise au cerveau par le nerf vague 
peut moduler le comportement sexuel (Wade et al 1996) mais aucune information n'est disponible en ce qui concerne la sécrétion du GnRH.

\section{b) En situation de déficit protéique}

Comme le rationnement énergétique, le rationnement protéique induit une réduction des concentrations plasmatiques d'insuline chez la truie en lactation. Il est donc possible que l'insuline (et le glucose) constitue une voie d'action commune aux deux types de déficits, dans l'interaction entre état métabolique et reproduction. L'influence du rationnement protéique sur IGF-I diffère selon les expériences : il réduit les concentrations circulantes d'IGF-I (Mejia-Guadarrama et al 2002, Quesnel et al 2005a) ou n'a pas d'effet significatif (Yang et al 2000b, Clowes et al 2003a et b).

Contrairement au déficit énergétique, le déficit protéique pendant la lactation ne s'accompagne pas d'une mobilisation accrue des lipides du tissu adipeux et les concentrations d'AGL et de leptine restent inchangées (MejiaGuadarrama et al 2002, Quesnel et al 2005a). Par contre, il s'accompagne d'une forte mobilisation des protéines corporelles, nettement plus importante qu'en cas de rationnement énergétique seul, et le profil plasmatique des AA est altéré. Or la sécrétion du GnRH est régulée par de nombreux peptides, dont la sérotonine, la dopamine et la noradrénaline, et certains de ces neurotransmetteurs nécessitent, pour leur synthèse, des AA précurseurs qui sont fournis exclusivement par les protéines alimentaires (AA essentiels). Ainsi, la sérotonine est synthétisée à partir du tryptophane et la dopamine directement à partir de la tyrosine ou indirectement à partir de la phénylalanine. Aussi peut-on se demander si la réduction de la disponibilité en certains AA pourrait constituer une voie d'action sur l'unité hy p ot hal a mo-hy poph y a i re. L'hypothèse a précédemment été évoquée chez la brebis (Miller et al 1998), car une suralimentation stimule la sécrétion de LH et simultanément induit une augmentation des concentrations de certains AA, notamment la tyrosine et la phénylalanine, dans le plasma et le liquide cérébrospinal (Miller et al 1998). Néanmoins, perfuser des brebis cycliques par du tryptophane, de la tyrosine ou un mélange de tyrosine et de phénylalanine est resté sans effet sur les sécrétions de LH, de FSH et d'autres hormones hypophysaires (GH et prolactine) (Downing et al 1997). Chez le porc en croissance, les concentrations en sérotonine dans le cerveau augmentent de façon dosedépendante lorsque la ration déficiente en tryptophane est enrichie en tryptophane purifié (Henry et al 1996). Le tryptophane, comme la tyrosine, sont des AA de haut poids moléculaire («Large Neutral AA», LNAA). Ils partagent un transporteur commun aux LNAA et leur captation par le cerveau dépend de la compétition avec les autres LNAA (Fernstrom 1983). Chez des cochettes prépubères, Barb et al (1997) observent que 48 heures de jeûne induisent une réduction du rapport tyrosine/LNAA dans le plasma. Ils suggèrent que cette réduction est à l'origine de l'augmentation des concentrations de GH observée chez ces femelles (les concentrations de LH ne sont pas altérées). Chez des truies rationnées en protéines pendant la lactation, nous avons mis en évidence une forte corrélation entre les concentrations de LH et le rapport tyrosine/LNAA ( $\mathrm{r}=-0,63, P<0,001$, Quesnel et al 2005b). D'autres travaux sont nécessaires pour déterminer si cette corrélation traduit une relation de cause à effet ou non.

\section{2 / Les médiateurs potentiels au niveau ovarien}

Dans de nombreuses espèces de mammifères, il a été montré que l'insuline et l'IGF-I jouent un rôle majeur dans la folliculogénèse. Elles stimulent la croissance et la maturation folliculaire, en partie en augmentant la réponse des cellules folliculaires aux hormones gonadotropes (Adashi et al 1985, Giudice 1992, Monget et al 1996). Chez la cochette cyclique, augmenter la concentration plasmatique d'insuline par injection ou par suralimentation augmente le taux d'ovulation, indépendamment des sécrétions gonadotropes (Cox et al 1987). L'insuline augmenterait le taux d'ovulation en diminuant l'atrésie folliculaire via une augmentation de l'IGF-I intrafolliculaire (Cox 1997). Des truies en déficit nutritionnel ou protéique présentent des concentrations intrafolliculaires d'IGF-I réduites au sevrage et dans les jours qui suivent, associées à une altération de la folliculogénèse (Quesnel et al 1998 a et b, Yang et al 2000b, Clowes et al 2003b). Les faibles concentrations d'insuline et d'IGF-I associées au déficit nutritionnel pendant la lactation affecteraient donc la folliculogénèse en réduisant la sensibilité des ovaires au signal gonadotrope induit par le sevrage des porcelets, d'une part, et en augmentant l'atrésie folliculaire avant l'ovulation, d'autre part.
Chez des truies rationnées en protéines pendant la lactation, une altération de la folliculogénèse peut être observée sans que les concentrations circulantes et intrafolliculaires d'IGF-I ne soient significativement altérées (Clowes et al 2003a, b). La folliculogénèse pourrait-elle être influencée par un déficit en AA circulants ? Jusqu'à présent, seules des corrélations ont été mises en évidence entre les concentrations circulantes de certains AA (notamment la méthionine) et le taux d'ovulation (Quesnel et al 2005b). Il serait intéressant d'étudier le rôle de la disponibilité en certains AA sur l'activité des cellules folliculaires.

\section{3 / L'interaction avec les réser- ves corporelles}

L'état des masses corporelles lipidiques et protéiques module la réponse de l'axe gonadotrope au déficit nutritionnel vraisemblablement parce qu'elles sont des réserves en substrats oxydables, mobilisables jusqu'à un certain point. Ainsi, les AGL servent de source alternative d'énergie, permettant une épargne du glucose. Les AA issus des protéines corporelles servent à la synthèse peptidique et protéique, mais sont aussi utilisables pour la néoglucogenèse. De ce fait, les réserves corporelles contribuent en partie à combler les déficits nutritionnels en atténuant leurs effets sur la reproduction. Ainsi, des truies qui pèsent $180 \mathrm{ou} 240 \mathrm{~kg}$ à la mise bas et qui sont rationnées en protéines alimentaires pendant la lactation présentent des profils d'AA différents au moment du tarissement (Quesnel et al 2005a). Il reste à déterminer si la mobilisation des protéines endogènes permet de maintenir un profil d'AA circulants compatible avec une activité hypothalamique suffisante, et ce d'autant plus que la masse musculaire est importante. Un poids vif élevé à la mise bas est aussi associé à des concentrations circulantes d'IGF-I plus élevées en fin de lactation (van den Brand et al 2001, Quesnel et al 2005a, figure 5). Cet effet sur l'IGF-I pourrait expliquer en partie l'effet protecteur des réserves sur les performances de reproduction, au moins au niveau ovarien.

\section{Conclusion}

Le déficit nutritionnel auquel peuvent être confrontées les jeunes truies pendant la lactation est toujours susceptible d'altérer les performances de reproduction après le sevrage, même si la réponse des truies semble avoir évolué 
Figure 5. Concentrations plasmatiques d'IGF-I aux alentours du sevrage (S) chez des truies primipares (Quesnel et al, données non publiées).

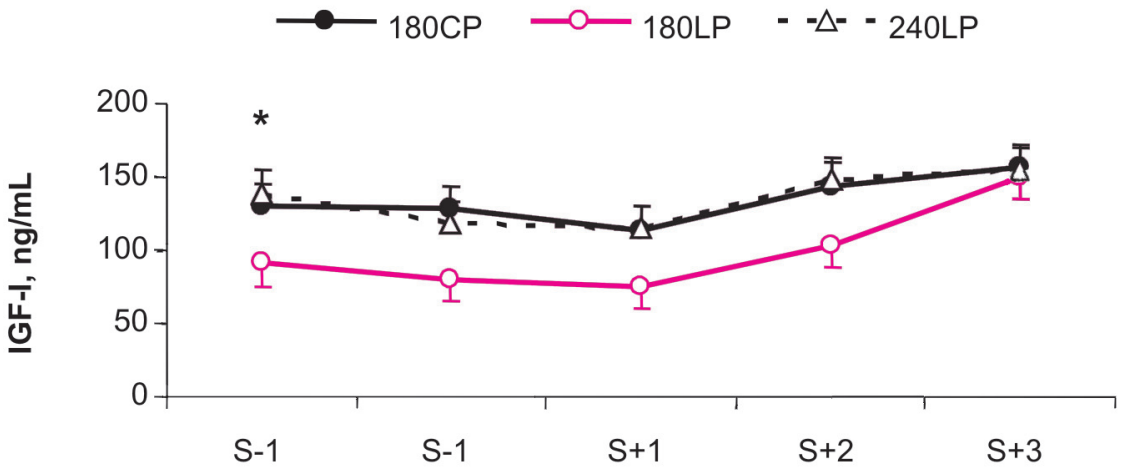

Jours par rapport au sevrage

${ }^{*} P<0,05$

Lots $180 \mathrm{CP}$ et $180 \mathrm{LP}$ : truies pesant environ $180 \mathrm{~kg}$ à la mise bas et qui sont rationnées (LP) ou non $(\mathrm{CP})$ en protéines pendant la lactation. Lot 240LP : truies pesant en moyenne $240 \mathrm{~kg}$ à la mise bas et rationnées en protéines pendant la lactation.

Figure 6. Représentation schématique des voies d'action par lesquelles le statut métabolique des truies en lactation pourrait influencer l'activité de l'axe gonadotrope.

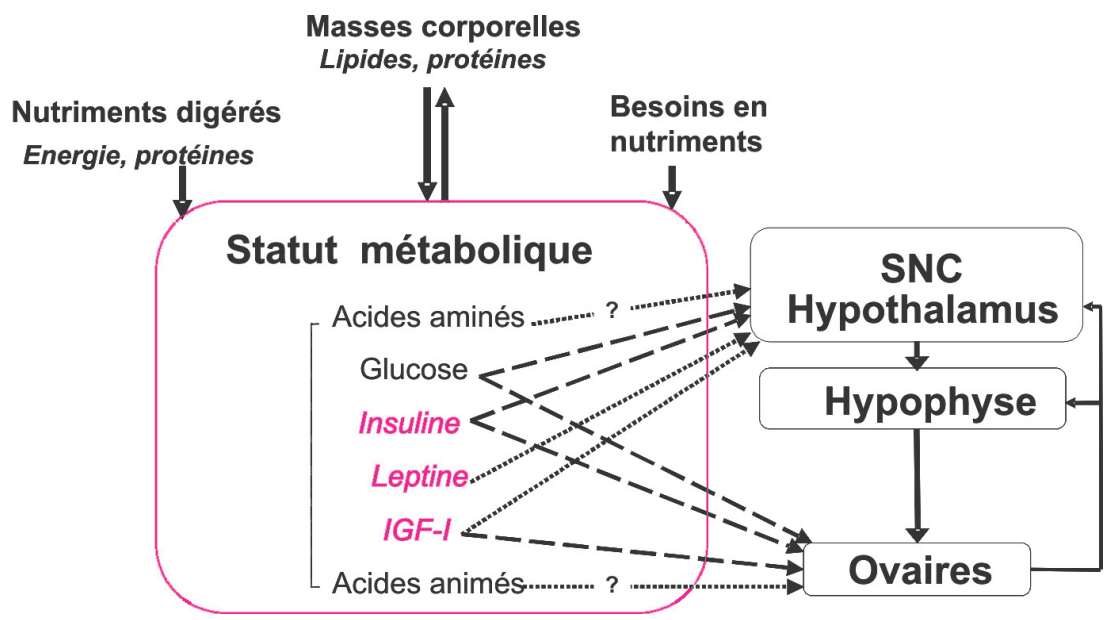

Les flèches en pointillés indiquent des voies d'action non étayées par la littérature chez la truie, ou qui mériteraient d'être étudiées plus avant (?). Les effets susceptibles de toucher directement l'hypophyse ne sont pas présentés ici. au cours de ces dernières années. En effet, un déficit sévère n'induit parfois qu'un retard marginal de l'œstrus après le sevrage, mais alors une dégradation du taux d'ovulation et/ou de la survie embryonnaire peut être observée. Cette dégradation pourra se répercuter sur la taille de la portée lorsque les conditions d'élevage sont globalement défavorables. Ces observations rappellent l'importance, en élevage, de bien maîtriser la conduite alimentaire des truies et leur état corporel. Dans le contexte actuel d'hyperprolificité, elles soulignent aussi le risque qu'il y a à laisser les jeunes truies allaiter des portées de grande taille.

L'activité de l'axe gonadotrope est touchée par le déficit nutritionnel à différents niveaux (figure 6). Les médiateurs potentiellement impliqués dans l'interaction entre statut métabolique et axe gonadotrope sont nombreux. Parmi eux, l'insuline et l'IGF-I joueraient un rôle majeur au niveau ovarien et l'insuline et le glucose au niveau hypothalamique. L'impact de la disponibilité de certains AA circulants sur l'activité hypothalamique et ovarienne mériterait d'être étudié de manière approfondie. Enfin, les mécanismes physiologiques par lesquels les réserves corporelles modulent l'impact du déficit nutritionnel sur l'axe gonadotrope restent à éclaircir.

\section{Remerciements}

L'auteur remercie Jean-Yves Dourmad pour son évaluation critique du manuscrit.

\section{Références}

Adashi E.Y., Resnick C.E., D’Ercole A.J., Svoboda M.E., Van Wyk J.J., 1985. Insulin-like growth factors as intraovarian regulators of granulosa cell growth and function. Endocr. Rev., 6, 400-420.

Aherne F.X., Kirkwood R.N., 1985. Nutrition and sow prolificacy. J. Reprod. Fert., Suppl., 33, 169-183.

Ahima R.S., Prabakaran D., Mantzoros C., Qu D., Lowell B., Maratos-Flier E., Filer J.S., 1996. Role of leptin in the neuroendocrine response to fasting. Nature, 382, 250-252.

Baidoo S.K., Aherne F.X., Kirkwood R.N., Foxcroft G.R., 1992. Effect of feed intake during lactation and after weaning on sow reproductive performance. Can. J. Anim. Sci., 72, 911-917.

Barb C.R., Kraeling R.R., Rampacek G.B., Dove C.R., 1997. Metabolic changes during the transition from the fed to the acute feed-deprived state in prepuberal and mature gilts. J. Anim. Sci., 75, 781-789.

Barb C.R., 1999. The brain-pituitary-adipocyte axis: role of leptin in modulating neuroendocrine function. J. Anim. Sci., 77, 1249-1257.

Barb C.R., Barrett J.B., Kraeling R.R. Rampacek G.B., 2001a. Serum leptin concentrations, luteinizing hormone and growth hormone secretion during feed and metabolic fuel restriction in the prepuberal gilt. Dom. Anim. Endocrinol., 20, 47-63.

Barb C.R., Kraeling R.R., Rampacek G.B., 2001b. Nutritional regulators of the hypothalamic-pituitary axis in pigs. Reproduction, Suppl., $58,1-15$.

Barb C.R., Kraeling R.R., 2004. Role of leptin in the regulation of gonadotropin secretion in farm animals. Anim. Reprod. Sci., 82-83, 155 167.

Boulot S., 2004. L'hyperprolificité en 2002 : quels résultats, quel impact sur la longévité des truies ? Journ. Rech. Porcine Fr., 36, 429-434.

Booth P.J., 1990. Metabolic influences on hypothalamic-pituitary-ovarian function in the pig. J. Reprod. Fert., Suppl. 40, 89-100.

Booth P.J., Cosgrove J.R., Foxcroft G.R., 1996. Endocrine and metabolic responses to realimentation in feed-restricted prepubertal gilts: associations among gonadotropins, metabolic hormones, glucose, and uteroovarian development. J. Anim. Sci., 74, 840-848.

Britt J.H., Armstrong J.D., Cox N.M. Esbenshade K.L., 1985. Control of follicular development during and after lactation in sows. J. Reprod. Fert., Suppl. 33, 37-54. 
Brüning J.C., Gautman D., Burks D.J., Gillette J., Schubert M., Orban P.C., Klein R., Krone W., Müller-Wieland D., Kahn C.R., 2000. Role of brain insulin receptor in control of body weight and reproduction. Science, 289, 21222125

Caraty A., Duittoz A., Pelletier J., Thiéry J.C., Tillet Y., Bouchard P. 2001. Libération pulsatile des gonadotropines, de la prolactine et de la GH. Le contrôle de la pulsatilité de LH. In : La reproduction chez les mammifères et l'homme, C. Thibault, M.C. Levasseur, INRA (eds), 85-107.

Charette R., Bigras-Poulin M., Martineau G.P., 1995. Une méta-analyse de l'anœestrus nutritionnel chez la truie. Journ. Rech. Porcine Fr., 27, 31-36.

Chilliard Y., Bonnet A., Delavaud C., Faulconnier Y., Leroux C., Djiane J., Bocquier F., 2001. Leptin in ruminants. Gene expression in adipose tissue and mammary gland, and regulation of plasma concentration. Dom. Anim. Endocrinol., 21, 271-295.

Clarke I.J., Henry B.A., 1999. Leptin and reproduction. Rev. Reprod., 4, 48-55.

Clowes E.J., Aherne F.X., Schaefer A.L., Foxcroft G.R., Baracos V.E., 2003a. Parturition body size and body protein loss during lactation influence performance during lactation and ovarian function at weaning in first-parity sows. J. Anim. Sci., 81, 1517-1528.

Clowes E.J., Aherne F.X., Foxcroft G.R., Baracos V.E., 2003b. Selective protein loss in lactating sows is associated with reduced litter growth and ovarian function. J. Anim. Sci., 81, 753-764.

Cosgrove J.R., Foxcroft G.R., 1996. Nutrition and reproduction in the pig: ovarian aetiology. Anim. Reprod. Sci., 42, 131-141.

Cox N.M., Stuart M.J., Althen T.G., Bennett W.A., Miller, H.W., 1987. Enhancement of ovulation rate in gilts by increasing dietary energy and administering insulin during follicular growth. J. Anim. Sci., 64, 507-516.

Cox N.M., Barb C.R., Kesner P.J., Kraeling R.R., Matamoros I.A., Rampacek G.B., 1990. Effects of intracerebroventricular administration of insulin on luteinizing hormone in gilts. J. Reprod. Fert., Suppl. 40, 63 (Abstract).

Cox N.M., 1997. Control of follicular development and ovulation rate in pigs. J. Reprod. Fert., Suppl., 52, 31-46.

Dagorn J., Le Cozler Y., Aumaître A., 1996. Incidence de la durée d'allaitement sur les performances de reproduction des truies dans les élevages français. Journ. Rech. Porcine Fr., 28, 287-294.

Dourmad J.Y., Etienne M., Prunier A., Noblet J., 1994. The effect of energy and protein intake of sows on their longevity: a review. Livest. Prod. Sci., 40, 87-97.

Dourmad J.Y., Noblet J., Etienne M., 1998. Effect of protein and lysine supply on performance, nitrogen balance and body composition changes of sows during lactation. J. Anim. Sci., $76,542-550$

Downing J.A., Scaramuzzi R.J., 1991. Nutrient effects on ovulation rate, ovarian function and the secretion of gonadotrophic and metabolic hormones in sheep. J. Reprod. Fert., Suppl. 43, 209-227.

Downing J.A., Joss J., Scaramuzzi R.J., 1997. Ovulation rate and the concentrations of $\mathrm{LH}$ $\mathrm{FSH}, \mathrm{GH}$, prolactin and insulin in ewes infused with tryptophan, tyrosine or tyrosine plus phenyalanine during the luteal phase of the oestrous cycle. Anim. Reprod. Sci., 45, 283-297.

Fernstrom J.D., 1983. Role of precursor availability in control of monoamine biosynthesis in brain. Physiol. Rev., 63, 484-546.

Foxcroft G.R., 1997. Mechanisms mediating nutritional effects on embryonic survival in pigs. J. Reprod. Fert., Supl. 52, 47-61.

Giudice L.C., 1992. Insulin-like growth factors and ovarian follicular development. Endocr. Rev., 13, 641-669.

Henry Y., Sève B., Mounier A., Ganier P., 1996. Growth performance and brain neurotransmitters in pigs as affected by tryptophan, protein and sex. J. Anim. Sci., 74, 2700-2710.

Houseknecht K.L., Portocarro, 1998. Leptin and its receptor: regulators of whole-body energy homeostasis. Dom. Anim. Endocrinol., 15, 457-475.

Hughes P.E., 1993. The effects of food level during lactation and early gestation on the reproductive performance of mature sows. Anim. Prod., 57, 437-445.

Jones D.B., Stahly T.S., 1999. Impact of amino acid nutrition during lactation on luteinizing hormone secretion and return to estrus in primiparous sows. J. Anim. Sci., 77, 1523-1531.

Kemp B., Soede N.M., Helmond F.A., Bosch M.W., 1995. Effects of energy source in the diet on reproductive hormones and insulin during lactation and subsequent estrus in multiparous sows. J. Anim. Sci., 73, 3022-3029.

King R.H., 1987. Nutritional anœstrus in young sows. Pig News Inf., 8, 15-22.

King R.H., Martin G.B., 1989. Relationships between protein intake during lactation, LH levels and oestrous activity in first-litter sows. Anim. Prod. Sci., 19, 283-292.

King R.H., Williams I.H., 1984. The effect of nutrition on the reproductive performance of first-litter sows. 2. Protein and energy intakes during lactation. Anim. Prod., 38, 249-256.

Kirkwood R.N., Baidoo S.K., Aherne F.X., Sather A.P., 1987. The influence of feeding level during lactation on the occurrence and endocrinology of the postweaning estrus in sows. Can. J. Anim. Sci., 67, 405-415.

Kirkwood R.N., Baidoo S.K., Aherne F.X., 1990. The influence of feeding level during lactation and gestation on the endocrine status and reproductive performance of second parity sows. Can. J. Anim. Sci., 70, 1119-1126.

Koketsu Y., Dial G.D., Pettigrew J.E., Marsh W.E., King V.L., 1996a. Influence of imposed feed intake patterns during lactation on reproductive performance and on circulating levels of glucose, insulin, and luteinizing hormone in primiparous sows. J. Anim. Sci., 74, 1036-1046.

Koketsu Y., Dial G.D., Pettigrew J.E., King V.L., 1996b. Feed intake pattern during lactation and subsequent reproductive performance of sows. J. Anim. Sci., 74, 2875-2884

Koketsu Y., Dial G.D., Pettigrew J.E., Xue J.L., Yang H., Lucia T., 1998. Influence of lactation length and feed intake on reproductive performance and blood concentrations of glucose, insulin and luteinizing hormone in primiparous sows. Anim. Reprod. Sci., 52, 153-163.

Le Cozler Y., Dagorn J., Dourmad J.Y., Johansen S., Aumaître A., 1997. Effect of weaning-to-conception interval and lactation length on subsequent litter size in sows. Livest. Prod. Sci., 51, 1-11.

Le Roith D., Bondy C., Yakar S., Liu J.L., Butler A., 2001. The somatomedin hypothesis: 2001. Endocrine Rev., 22, 53-74.

Lin J., Barb C.R., Matteri R.L., Kraeling R.R., Chen X., Meinersmann R.J., Rampacek G.B., 2000. Long form leptin receptor mRNA expression in the brain, pituitary, and other tissues in the pig. Dom. Anim. Endocrinol., 19, 53-61.

Lucia T, Dial G.D., Marsh W.E., 2000. Lifetime reproductive performance in female pigs having distinct reasons for removal. Livest. Prod. Sci., 63, 213-222.

Matamoros I.A., Cox N.M., Moore A.B., 1991. Effects of exogenous insulin and body condition on metabolic hormones and gonadotropin-induced follicular development in prepubertal gilts. J. Anim. Sci., 69, 2081-2091.

Mejia-Guadarrama C.A., Pasquier A. Dourmad J.Y., Prunier A., Quesnel H., 2002. Protein (lysine) restriction in primiparous lactating sows: effects on metabolic state, somatotropic axis, and reproductive performance after weaning. J. Anim. Sci., 80, 3286-3300.

Miller D.W., Blache D., Boukhliq R., Curlewis J.D., Martin G.B., 1998. Central metabolic messengers and the effects of nutrition on gonadotrophin secretion in sheep. J. Reprod. Fert., 112, 347-356.

Monget P., Besnard N., Huet C., Pisselet C. Monniaux D., 1996. Insulin-like growth factorbinding proteins and ovarian folliculogenesis. Human Res., 45, 211-217.

Monget P., Martin G.B., 1997. Involvement of insulin-like growth factors in the interactions between nutrition and reproduction in female mammals. Human Reprod., 12, Suppl. 1, 33-52.

Monget P., Etienne M., Rosetta L., 2001. Métabolisme énergétique et reproduction. In: La reproduction chez les mammifères et l'homme. INRA (eds), Paris, France, 749-769.

Mullan B.P., Williams I.H., 1989. The effect of body reserves at farrowing on the reproductive performance of first-litter sows. Anim. Prod., 48, 449-457.

Mullan B.P., Close W.H., Foxcroft G.R., 1991. Metabolic state of the lactating sows influences plasma LH and FSH before and after weaning. In: Manipulating Pig Production III, E.S. Batterham (ed.), Australasian Pig Science Association, Victoria, Australie, 32.

Noblet J., Dourmad J.Y., Etienne M., 1990 Energy utilization in pregnant and lactating sows: modeling of energy requirements. J. Anim. Sci., 68, 562-572.

Pettigrew J.E., Tokach M.D., 1993. Metabolic influences on sow reproduction. Pig News Inf., $14,69-72$.

Prunier A., Dourmad J.Y., Etienne M., 1993. Feeding level, metabolic parameters and reproductive performance of primiparous sows. Livest. Prod. Sci., 37, 185-196.

Prunier A., Quesnel H., 2000a. Nutritional influences on the hormonal control of reproduction in female pigs. Livest. Prod. Sci., 63, 1-16.

Prunier A., Quesnel H., 2000b. Influence of the nutritional status on ovarian development in female pigs. Anim. Reprod. Sci., 60-61, 185197.

Quesnel H, Prunier A., 1995. L'ovulation après le tarissement des truies : mécanismes 
physiologiques et facteurs de variation. INRA Prod. Anim., 8, 165-176.

Quesnel H., Pasquier A., Mounier A.M., Prunier A., 1998a. Influence of feed restriction during lactation on gonadotropic hormones and ovarian development in primiparous sows. J. Anim. Sci., 76, 856-863.

Quesnel H., Pasquier A., Mounier A.M Louveau I., Prunier A., 1998b. Influence of feed restriction in primiparous lactating sows on body condition and metabolic parameters. Reprod. Nutr. Dev., 38, 261-274

Quesnel H., Mejia-Guadarrama C.A., Dourmad J-Y., Farmer C., Prunier A., 2005a. Dietary protein restriction during lactation in primiparous sows with different live weight at farrowing: I. Consequences on sow metabolic status and litter growth. Reprod. Nutr. Dev., 45, 39-56.

Quesnel H., Mejia-Guadarrama C.A., Pasquier A., Dourmad J-Y., Prunier A., 2005b. Dietary protein restriction during lactation in primiparous sows with different live weight at farrowing: II. Consequences on reproductive performance and interactions with metabolic status. Reprod. Nutr. Dev., 45, 57-68.

Quiniou N., Dourmad J.Y., Noblet J., 1998. Facteurs de variation de l'appétit des truies en lactation. INRA Prod. Anim., 11, 247-249.

Shaw H.J., Foxcroft G.R., 1985. Relationships between LH, FSH and prolactin secretion and reproductive activity in the weaned sow. J. Reprod. Fertil., 75, 17-28.

Soede N.M., Prunier A., Kemp B., Quesnel H., 2000. Variation in weaning-to-œstrus interval in sows: causes and consequences. Reprod. Dom. Anim., Suppl. 6, 111-117.

Tokach M.D., Pettigrew J.E., Dial G.D., Wheaton J.E., Crooker B.A., Johnston L.J., 1992a. Characterization of luteinizing hormone secretion in the primiparous lactating sow: relationship to blood metabolites and return-toestrus interval. J. Anim. Sci., 70, 2195-2201.
Tokach M.D., Pettigrew J.E., Dial G.D., Wheaton J.E., Crooker B.A., Koketsu Y., 1992b. Influence of glucose infusions on luteinizing hormone secretion in the energy-restricted primiparous lactating sow. J. Anim. Sci., 70, 22022206.

van den Brand H., Dieleman S.J., Soede N.M., Kemp B., 2000. Dietary energy source at two feeding levels during lactation of primiparous sows. I. Effects on glucose, insulin, and LH and on follicle development, weaning-to-estrusinterval and ovulation rate. J. Anim. Sci., 78, 396-404.

van den Brand H., Prunier A., Soede N.M., Kemp B., 2001. In primiparous sows, plasma insulin-like growth factor-I can be affected by lactational feed intake and dietary energy source and is associated with luteinizing hormone. Reprod. Nutr. Dev., 41, 27-39.

Varley M.A., Foxcroft G.R., 1990. Endocrinology of the lactating sows. J. Reprod. Fert., Suppl. 40, 47-61.

Varley M.A., Prime G.R., Symonds H.W., Foxcroft G.R., 1996. Influence of food intake on plasma LH concentrations in multiparous sows. Anim. Reprod. Sci., 41, 245-253.

Vesseur P.C., Kemp B., Den Hartog L.A., 1994. The effect of weaning to estrus interval on litter size, live born piglets and farrowing rate in sows. J. Anim. Physiol. Anim. Nutr., 71, 30-38.

Wade G.N., Schneider J.E., Li H-Y, 1996. Control of fertility by metabolic cues. Am. J. Physiol., 270, E1-E19.

Wade G.N., Jones J.E., 2003. Lessons from experimental disruption of estrous cycles and behaviours. Med. Sci. Sports Exerc., 35, 15731580 .

Welt C.K., Chan J.L., Bullen J., Murphy R., Smith P., De Paoli A.M., Karalis A., Mantzoros C.S., 2004. Recombinant human leptin in women with hypothalamic amenorrhea. New England J. Med., 351, 987-997.

Whittemore C. T., Morgan C.T., 1990. Model components for the determination of energy and protein requirements for breeding sows: a review. Livest. Prod. Sci., 26, 1-37.

Williams I.H., Mullan B.P., 1989. Nutritional influences on sows. In: Manipulating pig production II. J.L. Barnett, D. P. Hennessy (eds), Australasian Pig Science Association, Victoria, Australie, 285-290.

Yang H., Foxcroft G.R., Pettigrew J.E., Johnston L.J., Shurson G.C., Costa A.N., Zak L.J., 2000a. Impact of dietary lysine intake during lactation on follicular development and oocyte maturation after weaning in primiparous sows. J. Anim. Sci., 78, 1001-1009.

Yang H., Pettigrew J.E., Johnston L.J., Shurson G.C., Wheaton J.E., White M.E., Koketsu Y., Sower A.F., Rathmachers J.A., 2000b. Effects of dietary lysine intake during lactation on blood metabolites, hormones, and reproductive performance in primiparous sows. J. Anim. Sci., 78, 1001-1009.

Zak L.J., Cosgrove J.R., Aherne F.X., Foxcroft G.R., 1997a. Pattern of feed intake and associated metabolic and endocrine changes differentially affect postweaning fertility in primiparous lactating sows. J. Anim. Sci., 75, 208-216.

Zak L.J., Xu X., Hardin R.T., Foxcroft G.R., 1997b. Impact of different patterns of feed intake during lactation in the primiparous sow on follicular development and oocyte maturation. J. Reprod. Fertil., 110, 99-106.

Zak L.J., Williams I.H., Foxcroft G.R., Pluske J.R., Cegielski A.C., Clowes E.J., Aherne F.X., 1998. Feeding lactating primiparous sows to establish three divergent metabolic states: I. Associated endocrine and postweaning reproductive performance. J. Anim. Sci., 76, 11451153 .

Zieba D.A., Amstalden M., Williams G.L., 2005. Regulatory roles of leptin in reproduction and metabolism: a comparative review. Dom. Anim. Endocrinol., 29, 166-185.

\section{Résumé}

Si le déficit nutritionnel que subissent la plupart des truies pendant la lactation est sévère, il peut résulter en un allongement de l'intervalle sevrage-œstrus, surtout chez les jeunes femelles. Le taux d'ovulation au premier œstrus post-sevrage peut également être réduit. Un déficit énergétique ou un déficit protéique induits expérimentalement ont les mêmes effets que le déficit nutritionnel global. L'impact du déficit nutritionnel sur les performances de reproduction peut être atténué si les réserves corporelles des truies sont élevées en début de lactation.

Le retard de l'ovulation après le sevrage des porcelets est dû à l'inhibition de la sécrétion pulsatile du GnRH par l'hypothalamus pendant la lactation. En revanche, la baisse du taux d'ovulation n'est pas nécessairement liée à l'altération des sécrétions gonadotropes, ce qui implique l'existence d'effets directs au niveau des ovaires. Les différents mécanismes par lesquels l'axe gonadotrope pourrait être informé des changements métaboliques subis par la truie sont passés en revue. La disponibilité en substrats oxydables, notamment en glucose, serait le premier signal qui informe l'hypothalamus du statut métabolique. L'insuline interviendrait en modulant la disponibilité en glucose au niveau cellulaire. L'influence de la leptine et de l'IGF-I sur la sécrétion du GnRH pendant la lactation n'est pas établie chez la truie. Enfin, certains acides aminés sont des précurseurs de neurotransmetteurs impliqués dans le contrôle de la sécrétion du GnRH, et leur moindre disponibilité pourrait participer à l'inhibition de LH pendant la lactation. Au niveau ovarien, la folliculogénèse peut être altérée par les faibles concentrations d'insuline et/ou d'IGF-I associées au déficit nutritionnel. L'influence d'autres facteurs, comme la disponibilité en certains acides aminés, ne peut pas être exclue. 


\begin{abstract}
Metabolic status and reproduction in the lactating sow

Many sows are submitted to a nutritional deficiency during lactation because of high nutrient requirements and insufficient feed consumption. When the nutritional deficiency is severe, it can result in a delayed oestrus after weaning, especially in young sows. Ovulation rate after weaning can also be altered. The same consequences are observed when sows are restricted only in energy or dietary protein. The consequences of the nutritional deficiency may be attenuated when sows have large body reserves in early lactation.

The delay of oestrus and ovulation after piglet weaning is mainly due to the inhibition of the episodic secretion of hypothalamic GnRH during lactation. In contrast, the reduction in ovulation rate may occur independently of changes in gonadotropin secretion, suggesting the existence of direct effects at the ovarian level. This review analyses the physiological mechanisms by which information on the metabolic changes is transmitted to the hypothalamus-pituitary-ovary axis. The availability of oxidisable metabolic fuels, such as glucose, could be the first signal informing the hypothalamus of the metabolic status. Insulin could play a role in modulating the availability of glucose in brain cells. The role of leptin and IGF-I on GnRH secretion during lactation has not yet been established in the sow. Lastly, some amino acids are necessary for the synthesis of neurotransmitters involved in the secretion of GnRH; a reduction in their availability could be involved in the inhibition of GnRH secretion during lactation. At the ovarian level, folliculogenesis is likely to be altered by the reduction in insulin and IGF-I concentrations induced by the nutritional deficiency. The influence of other factors, such as the availability of some amino acids, must not be ruled out.
\end{abstract}

H. QUESNEL H., 2005. Etat nutritionnel et reproduction chez la truie allaitante. INRA Prod. Anim., 18, 277-286. 